
allemande

49-1 | 2017

Berlin 1957-1994

\title{
Les médias à Berlin, au centre des conflits
}

\section{Valérie Robert}

\section{CpenEdition}

\section{Journals}

Édition électronique

URL : https://journals.openedition.org/allemagne/529

DOI : 10.4000/allemagne.529

ISSN : 2605-7913

Éditeur

Société d'études allemandes

\section{Édition imprimée}

Date de publication : 16 juin 2017

Pagination : 117-131

ISSN : 0035-0974

\section{Référence électronique}

Valérie Robert, "Les médias à Berlin, au centre des conflits », Revue d'Allemagne et des pays de lanque allemande [En ligne], 49-1 | 2017, mis en ligne le 16 juin 2018, consulté le 02 juin 2022. URL : http:// journals.openedition.org/allemagne/529 ; DOI : https://doi.org/10.4000/allemagne.529 


\section{Les médias à Berlin, au centre des conflits}

\section{- Valérie Robert*}

Berlin, ville vitrine, ville sous cloche, ville d'observation de l'autre, de l'ennemi, où les conflits sont exacerbés par une situation insulaire: toutes ces images valent aussi pour les médias berlinois, pris dans les conflits Est et Ouest mais aussi dans des luttes spécifiques aux sociétés respectives et dans un entrelacs de relations toujours interdépendantes et parfois difficiles à démêler. Moyens de communication, les médias permettent que celle-ci ait lieu entre chaque côté de la ville et son "arrièrepays» respectif mais aussi entre Berlin-Est et Berlin-Ouest, et ce dans les deux sens. Il s'agit d'une communication compliquée, parfois indirecte, à épisodes, mais qui existe, qui entraîne des évolutions et des dynamiques dans les médias eux-mêmes, et qui sert aussi à construire une image de l'autre société par le biais de l'observation de ses médias ${ }^{(1)}$.

Berlin n'est donc pas séparée en deux zones de communication et n'est pas non plus une zone de communication séparée du reste des pays dont elle relève; l'affrontement Est/Ouest se fait par le biais des médias dans la ville et par la ville, celle-ci devenant elle-même dans son organisation et dans sa matérialité un acteur et une chambre d'écho de ces conflits.

\section{Deux espaces de communication imbriqués}

Berlin est historiquement un centre de presse, mais ce marché a été fortement réduit pendant la période nazie. Dans l'après-guerre, les zones occidentales représentent un marché local et largement coupé du reste de l'Allemagne. Berlin-Ouest

* Maître de conférences en études germaniques, Université Sorbonne Nouvelle-Paris 3 / CEREG (Centre d'études et de recherches sur l'espace germanophone).

1 Jens Ruchatz, «Unsere Medien/ Eure Medien. Zur Logik und Geschichte deutsch-deutscher Medienbeobachtung», in: Thomas Beutelschmidt, Rüdiger Steinlein, Henning Wrage (éd.), Das literarische Fernsehen. Beiträge zur deutsch-deutschen Medienkultur, Francfort-sur-le-Main, Peter Lang, 2007, p. 156. 
reste cependant un marché très dense, avec un grand nombre de quotidiens, mais dont beaucoup vont disparaître pour des raisons économiques pendant la période étudiée. Les éditeurs de presse de Berlin-Ouest n’ont pas la possibilité d'étendre géographiquement leur diffusion $^{(2)}$ et leur audience est uniquement locale: les horaires des trains ne permettent pas une diffusion à temps dans le reste de la RFA, alors que l'inverse est vrai ${ }^{(3)}$. C'est la raison pour laquelle Berlin ne connaît plus de quotidien suprarégional - jusqu'à la création de la tageszeitung en 1979. Les journaux importants pour le débat public en RFA ne sont donc pas des journaux qui proviennent de Berlin. Le marché berlinois est de plus très sensible aux fluctuations économiques et politiques: ainsi, après la crise de 1958, plusieurs grands annonceurs renoncent à y faire de la publicité ${ }^{(4)}$. La concurrence de la télévision est très forte à cause de la situation insulaire, ce qui vaut autant pour l'audience que pour les recettes publicitaires $^{(5)}$. À la baisse des recettes publicitaires vient s'ajouter une baisse de la diffusion : entre 1960 et 1965, alors que la presse quotidienne de RFA augmente sa diffusion de $17 \%$, celle de Berlin-Ouest baisse de $7 \%{ }^{(6)}$. Plusieurs quotidiens disparaissent malgré les subventions fédérales ${ }^{(7)}$, et le marché est de plus en plus concentré: en 1959, le groupe Springer, alors basé à Hambourg, rachète la Berliner Morgenpost au groupe Ullstein, et absorbe l'ensemble du groupe en 1960, ce qui lui confère une position ultra-dominante. En 1989, les journaux Springer représentent plus des trois quarts de la diffusion des quotidiens de Berlin-Ouest. Il existe cependant quelques avantages,

Tableau 1 : Quotidiens à Berlin (1957-1989)

\begin{tabular}{|c|c|}
\hline Berlin-Ouest & Berlin-Est \\
\hline 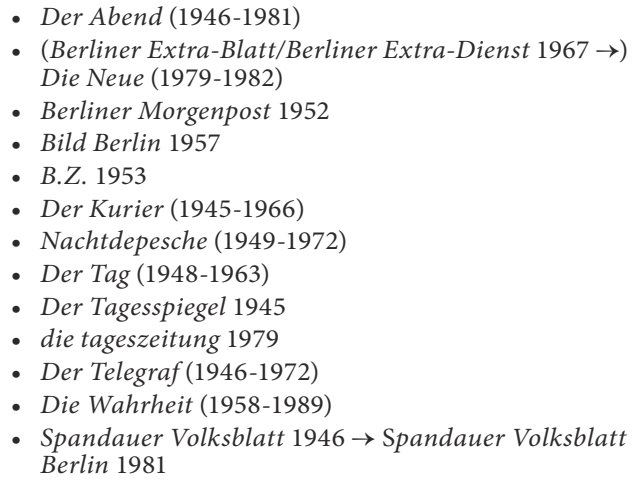 & $\begin{array}{l}\text { - Berliner Zeitung } 1945 \\
\text { - BZ am Abend } 1949 \\
\text { - Neues Deutschland } 1946 \\
\text { - } 8 \text { quotidiens suprarégionaux des Block- } \\
\text { parteien et des organisations de masse }\end{array}$ \\
\hline
\end{tabular}

2 Cf. Günter Bentele, Otfried Jarren, Ulrich Kratzsch (éd.), Medienlandschaft im Umbruch. Medien- und Kommunikationsatlas Berlin, Berlin, VISTAS, 1990, p. 11.

3 Peter de Mendelssohn, Zeitungsstadt Berlin. Menschen und Mächte in der Geschichte der deutschen Presse, Francfort-sur-le-Main/Berlin/Vienne, Ullstein, 1982 (éd. augm.), p. 583.

4 Ibid.

5 Ibid., p. 585.

6 Ibid.

7 Ibid., p. 592-595. 
par exemple les subventions fédérales spécifiques à Berlin grâce au programme de soutien financier du Bund (Berlinförderung) ${ }^{(8)}$. Il y a de plus à Berlin toute une série d'antennes et de succursales de ministères et d'administrations fédérales ${ }^{(9)}$ dont les personnels constituent un lectorat captif.

Il est en revanche impossible depuis la fin des années 40 de diffuser à l'Est la presse de l'Ouest $^{(10)}$, mais il y a encore en août 1961, lors de la construction du Mur, 52000 Berlinois de l'Est qui travaillent à l'Ouest et y achètent la presse, qui est aussi distribuée gratuitement par exemple lors de manifestations culturelles ${ }^{(11)}$. La construction du Mur interrompt cette diffusion ${ }^{(12)}$, ce qui aura des conséquences à la fois économiques pour les éditeurs de presse mais aussi sur le comportement des consommateurs de l'Est, qui vont largement se reporter sur l'audiovisuel de l'Ouest.

Du côté Est, les médias relèvent du système centralisé et contrôlé de RDA, dans lequel ils doivent suivre les consignes de la Abteilung Agitation du comité central du SED et, pour la presse, du Presseamt qui en dépend. Hormis Neues Deutschland et les journaux des organisations de masse et des partis, on trouve à Berlin-Est, la Berliner Zeitung et $B Z$ am Abend, qui dépendent de fait du SED ${ }^{(13)}$.

Pour autant, il faut se garder d'en conclure que la RDA était un système dans lequel les médias étaient totalement subordonnés aux intérêts du régime, et éviter de surestimer le contrôle de la population par les médias ${ }^{(14)}$. Les recherches récentes sur les médias de RDA ont montré que leurs contenus ont fait l'objet d'un «processus de négociation entre la société et les dirigeants, lesquels ont reculé progressivement et n'ont pas réussi à maintenir le contrôle de l'opinion publique ni une séparation rigide vis-à-vis de l'Ouest » ${ }^{(15)}$. Ceci est dû, bien sûr, à la présence de l'audiovisuel de l'Ouest dans le quotidien des habitants de l'Est, particulièrement sensible à Berlin, un phénomène d'influence et de transfert transnational qui relève de l'histoire croisée.

La plupart des programmes radio que les habitants de Berlin pouvaient écouter avaient été mis en place dès 1945/46, dont certains spécifiquement pour Berlin, ce qui montre l'importance de ce bassin d'auditeurs pour les différentes puissances

8 Stephan WeIchert, Leif Kramp, Der Berliner Pressemarkt. Historische, ökonomische und international vergleichende Marktanalyse und ihre medienpolitischen Implikationen, Berlin, Senatsverwaltung für Wirtschaft, Technologie und Frauen/Projekt Zukunft, 2009, p. 12.

9 Bernd Stöver, Geschichte Berlins, Munich, Beck, 2010, p. 84.

10 Franziska Kuschel, Schwarzhörer, Schwarzseher und heimliche Leser. Die DDR und die Westmedien, Göttingen, Wallstein, 2016, p. 49.

11 Ibid.

12 On peut à partir de 1977 acheter la presse occidentale à l'hôtel Metropol de Berlin-Est, mais en devises, ibid., p. 270.

13 Arne Kapitza, «Verlegerische Konzentration und redaktionelle "Ostalgie”: Die Printmedien", in: Roland Czada, Gerhard Leнмвruch (éd.), Transformationspfade in Ostdeutschland. Beiträge zur sektoralen Vereinigungspolitik, Francfort-sur-le-Main/New York, Campus, 1998, p. 250.

14 Stefan Zahlmann, "Medien in der DDR. Medienproduktion und Medienrezeption als kulturelle Praktiken", in: S. Zahlmann (éd.), Wie im Westen, nur anders. Medien in der DDR, Berlin, Panama Verlag, 2010, p. 17.

15 Christoph Classen, «DDR-Medien im Spannungsfeld zwischen Gesellschaft und Politik», in: ibid., p. 405. 
Tableau 2: Radio à Berlin 1957-1989

\begin{tabular}{|c|c|}
\hline Berlin-Ouest & Berlin-Est \\
\hline $\begin{array}{l}\text { - } \text { RIAS } 1946 \\
\text { - } R I A S 21953 \text {, devient un programme pour } \\
\text { jeunes en } 1985 \\
\text { - } \text { SFB } 1953 \rightarrow \text { SFB } 1,2,3 \\
\text { - Programme en allemand de la BBC } \\
\text { - Radios des forces alliées pour leurs soldats } \\
\text { respectifs } \\
\text { - À partir de } 1986 \text { radios privées } \\
\text { - Radios de l'opposition est-allemande émettant } \\
\text { de Berlin-Ouest: Roter Stachel } 1983-84 \text { et } \\
\text { Schwarzer Kanal } 1986^{(16)}\end{array}$ & $\begin{array}{l}\text { - Berliner Rundfunk 1946, Berliner Welle } 1958 \text { - } \\
1971 \\
\text { - Deutschlandsender } 1948 \rightarrow \text { Stimme der DDR } \\
1971 \\
\text { - Radio DDR I } 1953 \\
\text { - Radio DDR II } 1958 \\
\text { - DT64 1986/87 } \\
\text { - Radio Berlin International } 1987 \text { (radio vers } \\
\text { l'étranger) } \\
\text { - Radios clandestines vers l'Ouest, camouflées } \\
\text { en radios de l'Ouest }\end{array}$ \\
\hline
\end{tabular}

alliées ${ }^{(18)}$. Côté Ouest, le RIAS (Radio im amerikanischen Sektor), qui est resté jusqu'à sa disparition sous contrôle américain mais était financé depuis la fin des années 60 par le Bundesministerium für innerdeutsche Beziehungen, se définissait lui-même comme «la voix libre du monde libre» ${ }^{(19)}$ dans un contraste explicite avec les radios de l'Est, où il était considéré par les autorités comme un «poison ». Il diffusait depuis avril 1949 des émissions spécialement destinées au public de l’Est, notamment «Berlin spricht zur Zone» ou l'émission satirique «Pinsel und Schorchel» mettant en scène deux fonctionnaires du $\mathrm{SED}^{(20)}$. Le deuxième programme du RIAS devint en 1985 un programme pour jeunes censé faire pièce à la concurrence des radios privées. Si le RIAS dominait l'audience à Berlin, ce n'était pas seulement à cause de l'information mais aussi pour son offre de divertissement ${ }^{(21)}$. Le Sender Freies Berlin a pour sa part débuté ses programmes autonomes en 1953, après une période de coopération avec le Nordwestdeutscher Rundfunk sous contrôle britannique. Côté Est, le Berliner Rundfunk (nous passons sur les différentes étapes dans l'organisation de la radio en

Tableau 3: Télévision à Berlin 1957-1989

\begin{tabular}{|l|l|}
\multicolumn{1}{|c|}{ Berlin-Ouest } & \multicolumn{1}{|c|}{ Berlin-Est } \\
\hline $\begin{array}{l}\text { - Deutsches Fernsehen (ARD) 1954 } \\
\text { - ZDF } 1963\end{array}$ & $\begin{array}{l}\text { Deutscher Fernsehfunk 1956 } \\
\text { Fernsehen der DDR 1972: } \\
\text { - SFB } 1958 \rightarrow \text { Drittes Programmes } \\
\text { Bremen, SFB } \\
\text { - RIAS TV 1988 } \\
\text { - Chaînes privées à partir de 1986: Sat.1 avec programme } \\
\text { régional; RTL; chaînes du câble }\end{array}$ \\
- Chaînes des forces alliées à destination de leurs soldats & \\
\hline
\end{tabular}

16 F. Kuschel, Schwarzhörer (note 10), p. 276.

17 Klaus Arnold, «Musikbox mit Volkserziehungsauftrag. Radio in der DDR I», in : S. ZAHLMANN (éd.), Wie im Westen (note 14), p. 314.

18 B. Stöver, Geschichte Berlins (note 9), p. 75.

19 F. Kuschel, Schwarzhörer (note 10), p. 52.

20 Ibid., p. 47.

21 C. Classen, «DDR-Medien» (note 15), p. 398. 
RDA avant 1957) avait également jusqu'en 1971 un deuxième programme, la Berliner Welle, destinée spécifiquement aux auditeurs berlinois de l'Ouest. La zone Est créa en 1948 le Deutschlandsender destiné aux zones occidentales, qui devint en 1971 la Stimme der DDR, absorbant au passage la Berliner Welle. S'ajoutaient à cela les deux programmes Radio DDR I et $I I$, avec de plus, à partir de 1986/1987, le programme pour jeunes DT64, lancé en réponse à RIAS 2.

La télévision s'est développée plus tard mais a vite acquis une position dominante dans la concurrence entre médias. Les téléspectateurs avaient le choix à l'Ouest entre les programmes de l'ARD, du $Z D F$, un programme régional du SFB et enfin RIAS $T V$ à partir de 1988 et différentes chaînes privées à partir de 1986. Côté Est, les programmes de RDA se limitaient aux deux chaînes du Fernsehfunk, devenu Fernsehen der DDR en 1972.

\section{Regarder, écouter, observer l'autre}

La communication entre l'Est et l'Ouest passe donc par l'audiovisuel, et ce dans les deux sens. Si à l'Ouest, on craint de moins en moins l'influence des médias de l'Est ${ }^{(22)}$, ceux-ci ne sont pas si largement méprisés que le veut la représentation traditionnelle. Ainsi, en 1959, un sondage d'audience montre que le Deutscher Fernsehfunk a des spectateurs dans les zones de RFA qui peuvent le recevoir ${ }^{(23)}$, et - en réaction à cela? - le groupe Springer lance en 1960 dans ses magazines radio-télé, dont Hör zu, une campagne de boycott des programmes de l'Est, qui ne sont plus publiés ${ }^{(24)}$. Or il s'avère que les magazines continuant à publier la grille des programmes de l'Est voient leur diffusion augmenter fortement... Finalement, Springer reviendra sur le boycott, entre autres après que le Tagesspiegel a décidé en 1964 de publier à nouveau les programmes du DFF, avec un texte d'accompagnement commandé à Uwe Johnson ${ }^{(25)}$. Il semble qu'il y ait donc bien eu une demande, tout particulièrement à Berlin.

Du côté de la RDA, la consommation des médias n'est pas marquée par un rejet absolu des médias officiels et une adoption totale et sans recul des médias de l'Ouest: des entretiens avec des habitants de l'ex-RDA ont montré qu'ils consommaient en masse les médias de RDA sans pour autant être dupes du système, entre autres pour se tenir au courant des évolutions politiques, pour apprendre à décoder le discours officiel du moment ${ }^{(26)}$. Il y avait plutôt une utilisation en parallèle et complémentaire des médias de l'Est et de l'Ouest ${ }^{(27)}$, cette dernière ayant tout d'abord été combattue et punie par le régime, avant d'être reconnue et tolérée dans les années 70, même

22 Jens Ruchatz, «Einleitung», in: Jens Ruchatz (éd.), Mediendiskurse deutsch/deutsch, Weimar, VDG, 2005, p. 9-10.

23 J. Ruchatz, «Orte und Anlässe der Beobachtung. Eine Einleitung», ibid., p. 32.

24 Christina Bartz, J. Ruchatz, «Kommunikationsanlaß Springer», ibid., p. 78.

25 J. Ruchatz, «Orte» (note 23), p. 28.

26 Voir notamment Michael Meyen, Anke Fiedler, «Totalitäre Vernichtung der politischen Öffentlichkeit? Tageszeitungen und Kommunikationsstrukturen in der DDR», in: S. Zahlmann (éd.), Wie im Westen (note 14), p. 35-59.

27 C. Classen, «DDR-Medien» (note 15), p. 400. 
si l'audiovisuel de l'Ouest continuait à être considéré comme «un poison venant des ondes » ${ }^{(28)}$. Les programmes de l'Ouest étaient principalement consommés à des fins de divertissement, sauf dans les moments de crise où l'on recherchait de manière ciblée une information fiable ${ }^{(29)}$. D'après Christoph Classen ${ }^{(30)}$, l'influence des médias de l'Ouest n'était pas directement politique, elle a plutôt mené à diffuser un modèle de société de consommation, et les programmes de l'audiovisuel de l'Est qui correspondaient aux besoins des habitants étaient tout aussi appréciés que ceux de l'Ouest. Cependant, la présence permanente des programmes de l'Ouest a mené à une évolution des programmes de l'Est, qui se sont adaptés à la concurrence ${ }^{(31)}$. Il y a donc bien eu une influence, même si elle était publiquement niée. L'audiovisuel de l'Ouest reste en effet la (contre)-référence permanente, explicite ou implicite, des médias de l'Est et du discours officiel sur ces médias (par exemple avec l'émission «Der Schwarze Kanal» à la télévision de 1960 à 1989), tant dans la réalité de la consommation que dans les représentations. La définition de la société socialiste se fait en permanence par contraste avec l'Autre, et le discours sur l'Ouest passe en grande partie par le discours sur ses médias, un discours qu'on peut donc qualifier de transnational et qui mène à des représentations croisées et imbriquées des médias respectifs. Les médias ainsi thématisés ne sont pas seulement ceux qui sont effectivement consommés à l'Est, mais aussi ceux qui ne peuvent pas l'être, notamment, et tout particulièrement à Berlin, le groupe de presse Springer. Springer est en effet omniprésent dans la réalité des médias, dans le discours des médias et enfin dans la réalité matérielle de la ville, qui est le lieu d'une véritable intersection ayant des effets et des répercussions autant dans le discours que dans les pratiques médiatiques ${ }^{(32)}$.

\section{Axel Cäsar Springer, acteur et objet omniprésent de la communication}

Springer, c'est, dans la période qui nous occupe, le premier groupe de presse quotidienne en Allemagne, propriétaire entre autres de Bild et de Die Welt, un groupe fondé à Hambourg mais qui, à l'encontre de la tendance générale, va installer en 1967 son siège à Berlin. Après avoir absorbé le légendaire groupe Ullstein en 1960, Springer devient le groupe le plus puissant sur le marché berlinois. Les raisons de ce déménagement à Berlin sont financières (subventions) tout autant que politiques ${ }^{(33)}$, et les deux aspects

28 Gunter Holzweissig, "Massenmedien in der DDR», in: Jürgen Wilke (éd.), Mediengeschichte der Bundesrepublik Deutschland, Bonn, Bundeszentrale für politische Bildung, 1999, p. 587; voir également Meyen/Fiedler, «Totalitäre Vernichtung der politischen Öffentlichkeit?» (note 26), p. 35-59 et Knut Hickethier, «Das Fernsehen der DDR», in: S. Zahlmann (éd.), Wie im Westen (note 14), p. 119-130.

29 F. Kuschel, Schwarzhörer (note 10), p. 46.

30 C. Classen, «DDR-Medien» (note 15).

31 Ibid., p. 401; Uwe Breitenborn, «Spurrinnen und Leitplanken des Programms. Genre- und Formatentwicklung im Deutschen Fernsehen Ost», in: S. Zahlmann (éd.), Wie im Westen (note 14), p. 140.

32 Sur la question de l'intersection et de l'histoire croisée, voir notamment Michael WERnER, Bénédicte ZimmermanN, "Beyond comparison: Histoire croisée and the challenge of reflexivity", History and Theory, 45 (2006), p. 38.

33 Jochen StaAdt, Tobias Voigt, Stefan Wolle, Feind-Bild Springer: ein Verlag und seine Gegner, Göttingen, Vandenhoeck \& Ruprecht, 2009, p. 18. 
sont liés: Springer vise le marché de l'Est à court et long terme, en misant sur une réunification proche. Springer intervient d'ailleurs dans le champ politique, notamment en soutenant Willy Brandt pour l'élection à la mairie de Berlin ${ }^{(34)}$, et aussi par un projet politique pour Berlin et pour l'Allemagne. Ainsi, il part en janvier 1958 pour Moscou présenter à Khrouchtchev son plan, celui d'une Allemagne réunifiée neutre, qui comprend la réunification de Berlin sous contrôle allié. Derrière ce programme précis ${ }^{(35)}$, il y a une volonté politique mais aussi un intérêt économique: celui d'avoir de nouveau un marché médiatique uni et de pouvoir donc pénétrer le marché de l'Est - d'ailleurs, Springer aurait, d'après un rapport de la Stasi, envisagé de diffuser à l'Est ses magazines Hör zu et Constanze ${ }^{(36)}$ et c'est aussi dans ce cadre qu'il faut voir ses projets de télévision privée dans les années 50. Après l'échec du voyage à Moscou, Springer passe à une attitude de rejet global de la RDA, que ses journaux nommeront entre guillemets «DDR" jusqu'en août 1989. On trouve également parmi les principes de la maison d'édition, adoptés en 1967, l'engagement pour la réunification et le rejet de la RDA.

Springer est donc au plus tard depuis 1958 un ennemi acharné et puissant du second État allemand. Mais l'hostilité existe également dans l'autre sens, si bien que l'on peut parler d'une sorte d'affrontement localisé à Berlin. Alors même que les journaux Springer sont, après la construction du Mur, introuvables en RDA, celle-ci mène plusieurs campagnes contre Springer, qui visent donc moins les médias eux-mêmes que celui qui, pour Berlin-Est, symbolise le «système» de Bonn, la manipulation et l'abrutissement du public, une continuité présumée entre le national-socialisme et la $\mathrm{RFA}^{(37)}$, le capitalisme monopolistique et les relations entre politique et argent (il n'est pas anodin que la RDA utilise les deux prénoms de Springer et l'appelle la plupart du temps «Axel Cäsar Springer » ${ }^{(38)}$ ), et enfin le monopole dans la formation de l'opinion. Ce dernier point peut surprendre de la part d'un régime contrôlant les médias, mais ce qui compte, c'est la société que ce monopole est censé aider à construire ${ }^{(39)}$. Cette campagne se manifeste entre autres par la forte présence de Springer parmi les médias de l'Ouest critiqués par le «Schwarzer Kanal», ainsi que par des ouvrages et brochures ou encore une série télévisée (Fernsehroman) entièrement consacrée à Axel Springer et diffusée entre 1968 et 1970 à la télévision est-allemande ${ }^{(40)}$.

Mais l'affrontement passe aussi par l'architecture, ce dont témoigne le monument édifié à Berlin-Est pour le soldat Reinhold Huhn en 1963, tué par Rudolf Müller qui avait creusé un tunnel depuis le terrain appartenant à Springer pour aller chercher sa famille à Berlin-Est et la ramener de l'autre côté. Le monument a été édifié en quelque sorte à l'ombre de l'immeuble Springer qui se dressait de l'autre côté du Mur, avec un message clair: pour la RDA, c'était Springer qui était derrière cette affaire ${ }^{(41)}$. La

41 StaAdt et al., Feind-Bild Springer (note 33), p. 38-40. 
RDA et Springer se parlent ainsi, à Berlin, par le biais de l'architecture; reproches et accusations sont mis en scène et se répondent de part et d'autre du Mur.

Pour autant, l'affrontement est mêlé d'admiration; ainsi, il semble qu'il y ait eu en RDA, vers 1976, des réflexions menées au plus haut niveau pour développer une sorte de Bild local. Et dans l'autre sens, la RDA a produit à Berlin un journal d'un genre tout à fait unique: la Neue Bild-Zeitung, une copie de Bild produite à l'Est et destinée exclusivement au public de l'Ouest, parue entre 1957 et 1963 ou 1965 selon les sources ${ }^{(42)}$. Il s'agissait, en imitant le ton racoleur de Bild, de mettre en lumière des scandales en RFA et de dénoncer la société capitaliste; ce journal, édité par le Nationalrat de la Nationale Front, l'organisation qui regroupait l'ensemble des partis et organisations de masse de RDA, n'affichait pas son origine mais celle-ci était identifiable par différents indices, dont l'utilisation du terme "Westmark». On observe donc comment, dans le cas particulier de Berlin et plus largement de la relation Est/Ouest pendant la Guerre froide $^{(43)}$, les objets médiatiques «ne doivent pas seulement être considérés dans leur relation les uns aux autres mais l'un à travers l'autre» et qu'il faut donc «examiner les processus complexes d'interaction et d'appropriation ${ }^{(44)}$.

Le signe le plus tangible de la place de Springer à Berlin était l'immeuble Springer, qui «a énervé l'Est et l'Ouest pendant plus de 20 ans » ${ }^{(45)}$. Édifié entre 1959 et 1966 dans l'ancien quartier de la presse de Berlin, dont les ruines ne furent déblayées qu'en $1957^{(46)}$, et inspiré par l'immeuble du Daily Mirror à Londres, cet immeuble devait faire $130 \mathrm{~m}$ de haut et a finalement vu sa taille réduite à cause de la nature du sol; il restait cependant le bâtiment le plus haut de Berlin après le Funkturm ${ }^{(47)}$. La construction du Mur eut lieu juste à côté, pendant les travaux, menant Springer à se demander s'il fallait continuer l'édification ou se limiter à achever la partie du bâtiment qui devait abriter l'imprimerie $^{(48)}$. Finalement, il décida de poursuivre pour édifier un siège abritant à la fois la fabrication technique et les rédactions de ses quotidiens berlinois. Résolument tourné vers l'Ouest, ce bâtiment devait être un «Phare de la liberté» ("Leuchtturm der Freiheit») selon les termes de Springer ${ }^{(49)}$. Pour la RDA, ce bâtiment symbolisait l'Ouest et le conflit.

42 Ibid., p. 52-73; voir par exemple le numéro de 1965 conservé au Deutsches Historisches Museum, https://www.deutsche-digitale-bibliothek.de/item/3BLDFS7IOESC2EH4C6JSEKJMBDAQN4E4, page consultée le 10.02.2017.

43 Cf. à ce sujet Simo Miknonen, Pia Korvunen, «Beyond the Divide: Introduction», in: S. Mikkonen, P. Koivunen (éd.), Beyond the Divide: Entangled Histories of Cold War Europe, New York, Berghahn, 2015, p. 1-19.

44 Jonathan Bignell, Andreas Fickers, «Introduction: Comparative European Perspectives on Television History», in: J. Bignell, A. Fickers (éd.), A European Television History, Londres, Wiley Blackwell, 2008, p. 11.

45 Stephanie Warnke-De Nobili, «Jerusalemer Straße/Ecke Kochstraße. Anmerkungen zur Lage von Axel Springers West-Berliner Verlagshaus», in: Günter Schlusche, Verena Pfeiffer-Kloss, Gabi Dolff-Bonekämper, Axel Klausmeier (éd.), Stadtentwicklung im doppelten Berlin. Zeitgenossenschaften und Erinnerungsorte, Berlin, Ch. Links, 2014, p. 18.

46 P. de Mendelssohn, Zeitungsstadt Berlin (note 3), p. 574.

47 Ibid., p. 576.

48 Ibid., p. 581.

49 Discours à Columbia du 3.5.1967 cité par Manuel Seitenbecher, Den deutschen “Cäsar” bezwingen. Die 1960er und die Kampagne gegen Springer, Marbourg, Tectum, 2008, p. 49. 
Deux exemples montrent l'importance symbolique accordée à cet immeuble par Berlin-Est: en 1969, la rumeur circula en RDA que les Rolling Stones allaient donner un concert sur le toit de l'immeuble Springer ${ }^{(50)}$, ce qui mena des jeunes de tout le pays à s'approcher du Mur à cet endroit-là, provoquant des heurts avec la police et des arrestations. Indépendamment du caractère hautement improbable d'un tel concert pour quiconque connaissait le groupe Springer, peu friand de jeunes chevelus, cet épisode montre une chose: la RDA avait tant projeté sur Springer et son immeuble que celui-ci en était devenu pour la jeunesse l'incarnation toute-puissante de l'Ouest. Cette représentation a également eu des conséquences architecturales. En face de l'immeuble Springer se trouvait l'immeuble de la société immobilière GSW (Gemeinnützige Siedlungs- und Wohnungsbaugesellschaft), sur le toit duquel était placé un dispositif lumineux permettant d'afficher des informations visibles de loin, et donc de Berlin-Est à qui elles étaient destinées. Ce dispositif émanait du Sénat de Berlin. Or, dans les archives de Berlin-Est, Staadt et al. ont retrouvé des documents témoignant de discussions pour neutraliser ce dispositif que l'on localisait - faussement - sur le toit de l'immeuble Springer ${ }^{(51)}$. Finalement, cela mena à la construction d'un nouveau quartier sur la Leipziger Straße, qui devait permettre de bloquer la vue sur l'immeuble Springer. Ces immeubles, nommés par les habitants «Springerdecker», ont ainsi été construits sur la base d'une représentation fausse: l'information ennemie émanait nécessairement de Springer, et la lutte contre celui-ci a donc laissé une trace matérielle et architecturale dans l'aménagement urbain. La circulation des représentations, leur va-et-vient par-delà (ou par-dessus) le Mur, va donc au-delà d'une influence directe.

\section{7-1968 : Berlin contre Springer}

Pour Staadt et al., «les batailles médiatiques de ces années sont très certainement un 'lieu de mémoire' allemand» ${ }^{(52)}$. On peut même avancer l'idée que ce lieu de mémoire est commun aux deux parties de Berlin, dans la mesure où elles furent toutes les deux impliquées, certes de manière différente, dans un conflit dont l'épicentre se trouvait à Berlin-Ouest: celui entre Springer et l'APO (Außerparlamentarische Opposition) et le mouvement étudiant entre 1967 et 1969, qui était aussi un conflit de générations dans la ville divisée et un écho de la confrontation Est-Ouest. Ce conflit, spécifique à la société de RFA, converge cependant avec les attaques de la RDA contre Springer.

À l'Ouest, les critiques envers la position dominante du groupe Springer étaient de plus en plus nombreuses depuis le début des années 60, avec comme axes principaux: la forte concentration de la presse en RFA, encore plus marquée à Berlin, le poids de Springer dans la formation de l'opinion, et enfin, même si ce n'était pas affiché, une concurrence entre éditeurs de presse. Ce n'est pas un hasard si le Spiegel et son éditeur Rudolf Augstein furent à la tête du mouvement anti-Springer (en particulier par une série d'articles intitulée "Geschichte und Analyse des Springer-Konzerns»

50 StAadt et al., Feind-Bild Springer (note 33), p. 191.

51 «Mögliche Gegenmaßnahmen gegen die im Bau befindliche Nachrichtenleuchtschrift auf dem Hochhaus des Springer-Konzerns vom 16. Oktober 1963 mit Anschreiben von Paul Verner an Albert Norden vom 17. Oktober 1963 ", cité par ibid., p. 8-9.

52 Ibid., p. 13. 
en $\left.1968^{(53)}\right)$. Springer était donc omniprésent dans le discours public des années 60, ce qui mena en 1967 à la mise en place d'une commission du Bundestag chargée d'examiner la concentration dans les médias («Günther-Kommission»). À Berlin même, dont les étudiants passaient déjà dans les années 50 pour très engagés ${ }^{(54)}$, l'APO était très active, portée en particulier par le Republikanischer Klub et le SDS (Sozialistischer Deutscher Studentenbund), et s'engageait contre la guerre au Vietnam et la politique des États-Unis. Les nombreuses manifestations ont suscité une forte couverture médiatique, en particulier dans les journaux Springer, dont le discours était particulièrement violent, parlant de «terreur», de «rouges», de "Krawallmacher» et appelant les autorités à «éliminer les fauteurs de troubles parmi les étudiants» ou à cesser de financer "ces singes aux cheveux longs » ${ }^{(55)}$. La tension atteint son apogée en juin 1967 avec l'assassinat de l'étudiant Benno Ohnesorg (dont la mort mènera à une radicalisation de nombreux étudiants) lors d'une manifestation contre la visite du shah d'Iran, puis avec l'attentat contre Rudi Dutschke le 11.04.1968, attaque perpétrée par un lecteur de Bild. Pour de nombreux étudiants, c'est «Springer qui a tiré» et Springer, dont les journaux avaient fait de Dutschke l'ennemi public numéro 1, est responsable de l'attentat. Cela conduit à une manifestation devant l'immeuble Springer, lors de laquelle des vitres sont brisées et des véhicules de livraison incendiés ${ }^{(56)}$ (il y avait déjà eu des jets de pierre contre l'immeuble en février 1968). Le lendemain de l'attentat, l'APO réunie établit une liste de revendications adressée au Sénat de Berlin dans laquelle on voit bien le lien entre contestation sociale et médias. En plus de la démission du Sénat, elle réclame: l'expropriation de Springer et la mise en place, pour gérer l'entreprise, d'un conseil composé d'ouvriers, d'employés, d'étudiants et d'élèves; la démocratisation de l'audiovisuel, en particulier la fin du contrôle américain sur le $R I A S$, et l'entrée de représentants de l'APO dans les conseils du $S F B^{(57)}$. En parallèle, l'APO tente de mettre en place un "tribunal Springer", opération pour laquelle on envisage même de faire venir Wolf Biermann de l'Est, demande formulée par écrit auprès du ministère de l'Intérieur de RDA par Peter Schneider en novembre $1967^{(58)}$.

L'APO à l'Ouest et la RDA ont donc un ennemi commun, et Springer est un facteur d'unité au moins comme «objet d'une observation» et comme «thème 'germano-allemand'» ${ }^{(59)}$. La convergence des campagnes contre Springer a suscité la question d'une éventuelle manipulation par Berlin-Est des mouvements de l'Ouest. Si le groupe Springer est, dans son travail de mémoire et d'amélioration de son image, très favorable à cette hypothèse, c'est moins le cas des historiens, même ceux dont le travail a été financé par le groupe Springer - ainsi, Staadt et al. en arrivent à la conclusion que «le

53 BARtz/Ruchatz, «Kommunikationsanlaß Springer» (note 24), p. 59.

54 B. StÖver, Geschichte Berlins (note 9), p. 106.

55 Weichert/Kramp, Der Berliner Pressemarkt (note 8), p. 31.

56 Henno Lohmeyer, Springer. Ein deutsches Imperium, Berlin, edition q im Quintessenz Verlag, 1992, p. 330-333.

57 «Forderungen der APO an Senat und Abgeordnetenhaus von Berlin, Westberlin, den 12.04.1968», in: Deutsche Geschichte 1962-1983, Bd. 1, hg. und kommentiert von Irmgard Wilharm, Francfort-sur-leMain, Fischer, 1985, p. 175-178. 
mouvement étudiant antiautoritaire n'a pas été téléguidé par la RDA » ${ }^{(60)}$ et parlent de convergence plus que de manipulation. S’il est avéré qu'il y a eu des rencontres à BerlinOuest entre représentants de la FDJ et du SDS ${ }^{(61)}$, les comptes-rendus montrent que les approches étaient différentes, même si l'ennemi était le même. La critique de Springer du côté de l'APO était plutôt inspirée par l'École de Francfort et son analyse des médias, de l'industrie culturelle et de la culture de masse ${ }^{(62)}$, et l'arrière-plan intellectuel n'était pas le même que celui de la RDA. Cependant, on en tire la même conclusion: «Enteignet Springer!», une revendication qui semble avoir été exprimée pour la première fois, même si c'était en d'autres termes, par Walter Ulbricht en avril $1966^{(63)}$. À l'Ouest, on trouve pour la première fois ce slogan en Une du Berliner Extra-Blatt du 13.05.1967: «Expropriez Axel Cäsar Springer!»(«Enteignet Axel Cäsar Springer!»). La présence du «Cäsar» est un indice qui signale une origine à l'Est, et en effet, l'histoire de ce journal montre comment se mêlent campagne de l'APO, concurrence entre éditeurs et campagne venant de RDA. En 1966, Rudolf Augstein avait envisagé de fonder un nouveau journal à Berlin, qui devait être résolument anti-Springer. Il s'est retiré du projet en 1967, pas convaincu par la qualité journalistique du projet, mais a financé une partie ce qui est devenu le Berliner Extra-Blatt. Celui-ci, qui se présentait comme le journal de l'APO à Berlin-Ouest, bénéficiait en réalité d'une aide financière et rédactionnelle de la Stasi, et avait dans sa rédaction 5 IM - dont deux travaillaient de surcroît pour le Verfassungsschutz ${ }^{(64)}$, un bon résumé de la situation complexe de Berlin.

\section{Berlin, creuset de médias alternatifs}

D’une manière générale, la critique des médias Springer menée par l'APO avait besoin elle aussi de médias; comme tout mouvement social, sa publicité passait par la mise en scène d'événements répondant aux besoins des médias traditionnels, avec lesquels existait un rapport de "symbiose compétitive»" ${ }^{(65)}$. Mais le mouvement a aussi développé à Berlin-Ouest ses propres médias alternatifs: tracts, affiches, journaux comme le Berliner Extra-Blatt mais aussi films - ce dont témoignent les travaux d'étudiants de la Deutsche Film- und Fernsehakademie, fondée à Berlin en $1966^{(66)}$. La Gruppe 3, qui regroupait les étudiants les plus politisés, s'est précisément penchée sur la question du documentaire en traitant le thème de Springer et de la concentration dans la presse. On voit ainsi dans Unsere Steine de Ulrich Knaudt (1968) ${ }^{(67)}$ comment est brisée une des vitres de l'immeuble Springer, derrière laquelle figure

60 StaAdt et al., Feind-Bild Springer (note 33), p. 143.

61 Ibid., p. 123-124.

62 Dominik Lachenmeier, «Die Achtundsechziger-Bewegung zwischen etablierter und alternativer Öffentlichkeit», in: Martin Klimke, Joachim Scharloth (éd.), 1968. Handbuch zur Kultur- und Mediengeschichte der Studentenbewegung, Stuttgart, Metzler, 2007, p. 68.

63 StaAdt et al., Feind-Bild Springer (note 33), p. 76.

64 Pour l'histoire détaillée, voir ibid. p. 94-98 mais aussi M. Seitenbecher, Den deutschen "Cäsar" bezwingen (note 49), p. 45-46.

65 D. Lachenmeier, «Die Achtundsechziger-Bewegung» (note 62), p. 62.

66 Cf. notamment https://dffb-archiv.de/editorial/dies-jenseits-bilder-film-politik-dffb-1966-1995, page consultée le 10.02.2017.

67 https://dffb-archiv.de/dffb/unsere-steine, page consultée le 10.02.2017. 
une photo d'Axel Springer en compagnie du vice-président américain Hubert Humphrey, puis l'immeuble progressivement effacé par un nuage de fumée (6'00 à 6’44); au début du film, une jeune femme lit ce que la $B . Z$. écrit sur la guerre au Vietnam puis enveloppe dans le journal une pierre qu'elle lance ensuite (3'09 à 4'11). Dans Ihre Zeitungen (1967) ${ }^{(68)}$, Harun Farocki met en parallèle les bombardements américains au Vietnam et le bombardement de la population de RFA par des paquets de journaux (12’05 à 12'25); Brecht die Macht der Manipulateure de Helke Sander est aussi consacré à Springer et à la manipulation de l'opinion ${ }^{(69)}$.

Si les années 70 sont marquées par l'éclosion partout en RFA d'une presse alternative, c'est particulièrement le cas à Berlin; fortement liée à l'opposition aux médias dominants ${ }^{(70)}$, on ne peut, à Berlin, la dissocier de la critique contre Springer. Berlin fournit également un milieu urbain particulier, marqué par le mouvement étudiant qui suscite de nouvelles formes d'expression médiatique ${ }^{(71)}$. Ainsi, les années 70 voient la création du magazine féministe Courage (1976-1984) ou encore de tip en 1972, une nouvelle formule de city-magazine qui va devenir un succès économique. Enfin, c'est à Berlin qu'est créée en 1979 la tageszeitung, émanation des mouvements alternatifs et des Bürgerinitiativen, qui va devenir le seul quotidien suprarégional installé à Berlin. Très marquée par la critique des médias (la Une de son premier numéro quotidien représente un clown en train de lancer un pavé sur lequel est inscrit «taz», un rappel entre autres des pierres lancées dans les années 60 contre l'immeuble Springer), la taz est un nouveau modèle de journal, sans éditeur, financé par ses lecteurs et administré par son personnel et qui se veut la voix d'une Gegenöffentlichkeit. Si la taz s'installe à Berlin, c'est aussi pour des raisons économiques et pour profiter des subventions fédérales spécifiques à Berlin (30000 marks par mois) ${ }^{(72)}$. D’abord situé à Wedding, le journal déménage en 1989 dans la Kochstraße, en face de l'immeuble Springer, où le conflit avec Springer continue d'un point de vue rédactionnel mais aussi urbanistique.

\section{Berlin, capitale d'un espace public divisé}

Après la chute du Mur, les systèmes médiatiques se rapprochent au sens où l'Est adopte le modèle occidental; dans le domaine de la presse, cela se fait au profit des éditeurs de l'Ouest qui investissent largement sur ce nouveau marché. C'est ainsi le cas pour le Berliner Verlag, éditeur de la Berliner Zeitung et de BZ am Abend, qui est racheté par Gruner+Jahr et Robert Maxwell en 1990, et ce avant même que la Treuhand ne mette en vente les entreprises d'État de RDA. Les phases du changement sont les suivantes ${ }^{(73)}$ : d'octobre 1989 à février 1990, les rédacteurs en chef des grands journaux de RDA changent et le SED se sépare de plusieurs titres. Durant cette période, de nombreux journaux sont créés, après l'appel à la liberté d'expression et de la presse

$68 \mathrm{https} / / / \mathrm{dffb}$-archiv.de/dffb/ihre-zeitungen, page consultée le 10.02.2017.

69 https://dffb-archiv.de/dffb/brecht-die-macht-der-manipulateure, page consultée le 10.02.2017.

70 Cf. Christina holtz-Bacha, «Alternative Presse», in: J. Wilke (éd.), Mediengeschichte der Bundesrepublik Deutschland (note 28), p. 330-349.

71 Bentele et al., Medienlandschaft im Umbruch (note 2), p. 191.

72 Weichert/Kramp, Der Berliner Pressemarkt (note 8), p. 12.

73 A. Kapitza, "Verlegerische Konzentration und redaktionelle “Ostalgie” (note 13), p. 242-244. 
exprimé notamment lors de la grande manifestation sur l'Alexanderplatz du 4.11.1989. Dans la phase suivante, à partir de mars 1990, le PDS, successeur du SED, arrête ses subventions aux journaux, qui sont contraints d'avoir recours à l'aide des éditeurs de l'Est, c'est notamment le cas pour le Berliner Verlag, qui se retrouve indépendant en avril 1990 et va donc trouver des investisseurs de l'Ouest. La troisième phase, qui dure jusqu'à l'été 1991, est marquée par la privatisation des titres menée par la Treuhand; la quatrième phase, qui débute au milieu de 1991, voit «le déclin et la disparition de la plupart des quotidiens suprarégionaux, des anciens journaux des Blockparteien, des créations de la Wende et d'une bonne partie de titres de magazines traditionnels » ${ }^{(74)}$. Dans l'audiovisuel, les chaînes et stations de radio de l'Est disparaissent en tant que telles et leurs fréquences sont intégrées dans le système existant, ce qui va aussi mener à des changements dans l'audiovisuel de Berlin-Ouest. Le RIAS, en particulier, disparaît en tant que tel avec la fin de la Guerre froide.

Tableau 4: Quotidiens à Berlin 1990-1994

\begin{tabular}{|c|c|}
\hline Berli & Berlin-Est \\
\hline $\begin{array}{l}\text { - Berliner Morgenpost } \\
\text { - Bild Berlin } \\
\text { - B.Z. } \\
\text { - Der Tagesspiegel (racheté par le groupe Holtz- } \\
\text { brinck en 1992) } \\
\text { - die tageszeitung } \\
\text { - Spandauer Volksblatt Berlin } \rightarrow \text { Volksblatt } \\
\text { Berlin } 1991 \\
\text { - Die Welt (Springer) déménage de Bonn à } \\
\text { Berlin } 1993\end{array}$ & $\begin{array}{l}\text { - BZ am Abend } \rightarrow 1990 \text { Berliner Kurier } \\
\text { am Abend } \rightarrow \text { Berliner Kurier am } \\
\text { Morgen } \rightarrow \text { Berliner Kurier (racheté en } 1990 \\
\text { par Gruner+Jahr/Maxwell, } 1992 \text { Gruner+Jahr } \\
\text { seul propriétaire) } \\
\text { - Berliner Zeitung (racheté en } 1990 \text { par } \\
\text { Gruner+Jahr/Maxwell, } 1992 \text { Gruner+Jahr seul } \\
\text { propriétaire) } \\
\text { - Neues Deutschland } \\
\text { - Privatisation par la Treuhand des journaux du } \\
\text { SED }\end{array}$ \\
\hline
\end{tabular}

Tableau 5 : Audiovisuel à Berlin 1990-1994 ${ }^{(75)}$

- SFB 1 télévision

- Radio DDR2, Stimme der DDR, RIAS $1 \rightarrow$ réunis dans Deutschlandsender Kultur 1990 puis Deutschlandradio (Berlin/Cologne) 1994

- Berliner Rundfunk privatisé 1992

- $R I A S-T V \rightarrow D W-T V 1992$

- RIAS 2 privatisé 1992

Le vote de 1991 en faveur du déménagement à Berlin a suscité l'espoir de voir Berlin redevenir une capitale de la presse, menant à une « course de vitesse des grands éditeurs allemands pour mettre en place un journal de la capitale» ${ }^{(76)}$, par exemple lorsque le groupe Holtzbrinck rachète le Tagesspiegel en 1992, mais ceci se situe en grande partie dans les années postérieures à la période étudiée ici. Berlin reste cependant le marché le plus difficile de RFA, et il a été impossible de développer un nouveau suprarégional

74 Ibid., p. 244.

75 Cf. particulièrement Bentele et al., Medienlandschaft im Umbruch (note 2), p. 260-261.

76 Sophie Mützel, «Von Bonn nach Berlin: Der gewachsene Hauptstadtjournalismus», in: Stephan Weichert, Christian Zabel (éd.), Die Alpha-Journalisten. Deutschlands Wortführer im Porträt, Cologne, Halem, 2007, p. 55, citée par Weichert/Kramp, Der Berliner Pressemarkt (note 8), p. 14. 
émanant de la capitale - même si le groupe Springer a déménagé Die Welt de Bonn à Berlin en 1993, suivi par la Welt am Sonntag en 2001 et par Bild en 2008 qui ont rejoint le reste des quotidiens dans l'immeuble Springer.

Les traces de la séparation, matérielle mais aussi en systèmes médiatiques différents, sont bien présentes dans les habitudes de consommation des médias: ainsi, on continue à lire davantage le Tagesspiegel à l'Ouest et la Berliner Zeitung à l'Est ${ }^{(77)}$, même si les deux journaux ont des lecteurs de l'autre côté, comme c'est aussi le cas pour la Berliner Morgenpost ou B.Z. La tageszeitung pour sa part reste un suprarégional de l'Ouest. La séparation Est/Ouest ne s'arrête d'ailleurs pas à Berlin, et le Mur continue à exister dans l'espace public ${ }^{(78)}$; c'est aussi le cas pour l'audiovisuel, les téléspectateurs des nouveaux Länder ayant une préférence pour les programmes de divertissement privés mais aussi pour les programmes régionaux qui traitent de leur actualité spécifique (ce qui n'est pas le cas des programmes nationaux) et rediffusent les émissions de divertissement cultes de $\mathrm{RDA}^{(79)}$. Enfin, alors même que les traces du Mur ont été effacées très rapidement, les conflits autour des médias ont laissé une trace matérielle dans la chair de la ville: ainsi, un morceau de la Lindenstraße et de la Kochstraße ont été rebaptisés respectivement en 1996 et en 2008, et se croisent désormais sous le nom de Axel-Springer-Straße et Rudi-Dutschke-Straße. Le groupe Springer a son siège au numéro 65 de la première, la tageszeitung a ses locaux au numéro 23 de la deuxième. Le croisement de ces deux rues raconte en concentré l'histoire des médias à Berlin, à l'intersection de deux systèmes et en permanente interaction les uns avec les autres, des médias qui ont investi l'espace urbain et l'ont ainsi transformé de manière durable.

\section{Résumé}

L'affrontement Est/Ouest se fait à Berlin par le biais des médias dans la ville et par la ville, celle-ci devenant elle-même dans son organisation et dans sa matérialité un acteur et une chambre d'écho de ces conflits. Les deux espaces de communication sont imbriqués, en particulier par la circulation des médias audiovisuels d'Ouest en Est mais aussi d'Est en Ouest. L'audiovisuel de l'Ouest est la (contre)-référence permanente, explicite ou implicite, des médias de l'Est et du discours officiel sur ces médias, tant dans la réalité de la consommation que dans les représentations. On a donc affaire à un phénomène transnational de représentations croisées et imbriquées des médias respectifs, qui fait qu'ils s'influencent également mutuellement. Le groupe Springer, installé à Berlin, est à la fois un acteur (notamment avec son immeuble de la Kochstraße) mais aussi un objet omniprésent de la communication et des conflits des deux côtés du Mur, ce qui aura des conséquences à moyen et long terme dans le domaine des médias (naissance de nouveaux médias alternatifs) mais aussi sur le plan de l'urbanisme.

77 Ibid., p. 90.

78 Valérie Robert, La presse en France et en Allemagne, Paris, Presses Sorbonne Nouvelle, 2012, p. 89-90.

79 Beate Schneider, «Massenmedien im Prozeß der deutschen Vereinigung», in: J. Wilke (éd.), Mediengeschichte der Bundesrepublik Deutschland (note 28), p. 621. 


\section{Zusammenfassung}

Der Ost-West-Konflikt hat in Berlin auch durch die Medien stattgefunden; die Stadt Berlin selbst ist in ihrer Organisation und ihrer Materialität zu einem Aktanten und einer Echokammer dieser Konflikte geworden. Die beiden Öffentlichkeiten bzw. Kommunikationsräume waren insbesondere dadurch miteinander verflochten, dass Rundfunkmedien von West nach Ost, aber auch umgekehrt sendeten. Der Westrundfunk blieb für die Ostmedien ein ständiges, implizites oder explizites Gegenmodell, was auch für den offiziellen Diskurs über diese Medien der Fall war; dies galt sowohl für die Medienkonsumgewohnheiten als auch für die Repräsentationen. Man hat es hier mir einem transnationalen Phänomen von verflochtenen Repräsentationen der jeweiligen Medien $z u$ tun, die in einem wechselseitigen Verhältnis stehen und dadurch auch einen Einfluss auf einander ausüben. Der seit den 60er Jahren in Berlin ansässige Springer-Konzern war mit seinem Springerhochhaus sowohl Aktant als auch allgegenwärtiges Objekt der über die Mauer hinweg stattfindenden Kommunikation und der konvergierenden bzw. mit einander verflochtenen Konflikte. Dies sollte mittel- und langfristig Spuren im Bereich der Medien (mit der Gründung neuer, alternativer Medien) wie auch im Stadtbild selbst hinterlassen. 